\title{
Perfil demográfico, clínico e funcional de idosas participantes e não-participantes de atividades comunitárias ligadas à igreja*
}

\section{Demographic, clinical and functional profile of elderly participants and non-participants in community activities of church}

\author{
Alline Meyre de Oliveira1, Costa, Patrícia Azevedo Garcia ${ }^{2}$
}

OLIVEIRA, A. M., GARCIA, P. A. Perfil demográfico, clínico e funcional de idosas participantes e não-participantes de atividades comunitárias ligadas à igreja. Rev. Ter. Ocup. Univ. São Paulo, v. 22, n. 2, p. 153-161, maio/ago. 2011.

RESUMO: Introdução: Atividades sociais e comunitárias são referidas na literatura científica como estratégias para promoção de saúde e de qualidade de vida no envelhecimento. Objetivo: Traçar e comparar o perfil demográfico, clínico e funcional de idosas participantes e não-participantes de atividades comunitárias ligadas à igreja. Métodos: Dezenove idosas, sendo 10 participantes e 9 nãoparticipantes de atividades ligadas à igreja, submeteram-se à avaliação sócio-demográfica, cognitiva (Mini-Exame do Estado Mental) e funcional (Timed Get Up and Go e Short Physical Performance Battery). Resultados: As idosas participantes destas atividades apresentaram um tempo médio de atuação de 23,62 anos. Essas idosas apresentaram idade menor, mais anos de estudo, melhor desempenho cognitivo, menor prevalência de doenças auto-relatadas, de quedas e de medo de cair, e melhor desempenho funcional quando comparadas às não-participantes. Observou-se correlação significativa entre anos de participação nas atividades com a idade $(\mathrm{r}=-0,579)$ e com o desempenho cognitivo $(r=0,467)$. Conclusão: As idosas participantes de atividades ligadas à igreja apresentaram perfil mais favorável ao envelhecimento ativo, incluindo melhor desempenho cognitivo e funcional, quando comparadas às não-participantes. A participação nessas atividades parece ser benéfica para manutenção da capacidade funcional para o envelhecimento ativo e saudável, e, desta forma, deve ser incentivada para complementar os programas de promoção de saúde e prevenção de doenças.

PALAVRAS-CHAVE: Idoso, Atividades cotidianas, Cognição; Participação comunitária; Promoção da saúde; Limitação da mobilidade.

*Este manuscrito é parte integrante de um trabalho de conclusão de curso de especialização em Fisioterapia em Geriatria e Gerontologia do Centro de Estudos Avançados e Formação Integrada - CEAFI.

1. Fisioterapeuta, especialista em Fisioterapia em Geriatria e Gerontologia - Centro de Estudos Avançados e Formação Integrada - CEAFI, Goiânia, GO, Brasil.

2. Mestre em Ciências da Reabilitação - Docente do curso de Fisioterapia do Colegiado de Fisioterapia da Universidade de Brasília - Campus Ceilândia - UnB, Brasília, DF, Brasil.

Endereço para corresepondência: Patrícia Azevedo Garcia. Área Especial 2, módulo F, torre III, apto 801, Ed. Via Boulevard, Bairro Guará II. Brasília - DF. CEP: 71.070-662 e-mail: patriciaagarcia@unb.br. 


\section{INTRODUÇÃO}

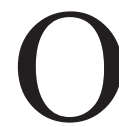

envelhecimento da população mundial é um dos grandes desafios a serem enfrentados no século XXI (GARRIDO; MENEZES, 2002)

e, no Brasil, a população com 60 anos ou mais já representa 8,6\% da população total (GARRIDO et al., 2002; COSTA; PORTO; SOARES, 2003). Esse processo de aumento da expectativa de vida vem acompanhado por modificações no perfil de saúde de sua população e predomínio de doenças crônicas, inatividade, declínios cumulativos em diversos sistemas fisiológicos, vulnerabilidade para condições de saúde adversas, dependência funcional, quedas, incapacidade física, alterações cognitivas, restrições de atividades e afastamento social (RAMOS et al., 1987; KILSZTAJN et al., 2003; CARVALHO, 2003; WONG; CARVALHO, 2006; FRIED et al., 2004; WALSTON et al., 2006; HECKMAN, 2006; SILVA et al., 2006). Essa realidade aponta para sérias repercussões sobre os aspectos sociais e econômicos (COSTA et al., 2003; CHAIMOWICZ, 1997; HECKMAN, 2006; FRIED et al., 2001, 2004) e reforça a complexidade crescente das alternativas de atenção às necessidades desta estrutura etária emergente (COSTA et al., 2003; CHAIMOWICZ, 1997).

Visando contemplar as necessidades da população idosa, o paradigma do "Envelhecimento Ativo", proposto pela Organização Mundial de Saúde, reforça a necessidade de otimização das oportunidades de saúde, participação e segurança, objetivando a melhoria da qualidade de vida dos idosos (DIAS et al., 2010; OMS, 2005). Neste sentido, destaca-se a importância da participação dos idosos em atividades sociais e comunitárias, incluindo os grupos de idosos nas igrejas e universidades, para obtenção do bemestar físico, mental e social (DIAS et al., 2010; CASTRO et al., 2007), tendo em vista que esses grupos oferecem programação social, atividades físicas, manuais, favorecem o engajamento das pessoas, facilitam a interação com novos amigos (SANTOS et al., 2009) e podem gerar impacto positivo no desempenho funcional dos idosos (AVLUND et al., 2002; ROSA et al, 2003).

Diante desta realidade, o objetivo do presente estudo foi traçar e comparar o perfil demográfico, clínico, cognitivo e funcional de idosas participantes e não-participantes de atividades comunitárias ligadas à igreja.

\section{PROCEDIMENTOS METODOLÓGICOS}

\section{éticos \\ Tipo de estudo, local do Eestudo e Aaspectos}

A pesquisa foi delineada como um estudo observacional transversal de natureza descritiva e exploratória, aprovada pelo Comitê de Ética em Pesquisa da Universidade Católica de Brasília (UCB) (Parecer 107/2010) e realizada nos domicílios das idosas voluntárias a participar do estudo, de junho a agosto de 2010. As participantes assinaram o Termo de Consentimento Livre e Esclarecido (TCLE).

\section{Amostra}

A amostra não-aleatória deste estudo foi constituída por 19 pessoas com 60 anos ou mais, residentes na comunidade, sendo 10 participantes e 9 não-participantes de atividades comunitárias ligadas à igreja. Essas atividades são realizadas por pessoas de diversas faixas etárias, porém com maior prevalência de idosos, e consistem em reuniões periódicas para desenvolvimento de trabalhos internos ou externos à igreja, tais como reuniões, visitas domiciliares, acompanhamento e assistência religiosa às famílias.

As participantes foram selecionadas por conveniência na Paróquia São João Bosco em Brasília - DF e para serem incluídas deveriam ter idade igual ou superior a 60 anos, ter mobilidade preservada com ou sem necessidade de dispositivo de auxílio à marcha, não ter praticado exercício físico regular nos últimos seis meses e ser participante ou não de atividades comunitárias ligadas à igreja. Foram excluídas idosas que apresentaram afasia, doenças crônicodegenerativas em estágio agudo ou em estágio avançado, sequelas motoras, déficits cognitivos detectáveis por meio de escores compatíveis com a escolaridade (BERTOLUCCI et al., 1994), déficits visuais e auditivos que impossibilitassem a realização dos testes ou dificultassem a compreensão do questionário clínico e sócio-demográfico.

\section{Instrumentos para coleta de dados}

Para avaliação da capacidade funcional foram utilizados o teste Timed Get Up and Go (TUG) e Short Physical Performance Battery (SPPB). O TUG caracterizase como um teste comumente utilizado para avaliar o controle postural dinâmico de residentes na comunidade e idosos frágeis, de fácil aplicação, realizado com o uso de calçado habitual e, se necessário, com dispositivo de auxílio (SHUMWAY-COOK et al., 2000). O SPPB é um instrumento traduzido e adaptado para população brasileira por Nakano (2007) composto por três testes que avaliam, sequencialmente equilíbrio estático em pé, velocidade de marcha em passo habitual e, indiretamente, força muscular 
OliveIRA, A. M., GARCIA, P. A. Perfil demográfico. Rev. Ter. Ocup. Univ. São Paulo, v. 22, n. 2, p. 153-161, maio/ago. 2011.

de membros inferiores. Para avaliação da capacidade cognitiva foi utilizado o Mini-exame do Estado Mental (MEEM), um instrumento que fornece informações sobre diferentes parâmetros cognitivos (BERTOLUCCI et al., 1994).

\section{Procedimentos}

Após serem contatadas na Paróquia, esclarecidas sobre os objetivos e procedimentos do estudo e aceitarem participar da pesquisa, as idosas assinaram o TCLE e foram agendadas as datas para avaliação. As participantes foram avaliadas, sempre pelo mesmo examinador, em seus próprios domicílios. Inicialmente foi realizada uma entrevista para registro das variáveis clínicas e sócio-demográficas (idade, procedência, anos de estudo, profissão anterior, estado civil, doenças existentes, histórico de quedas, medo de cair, hábitos de risco e tempo de participação em atividades comunitárias ligadas à igreja) e para determinação do nível cognitivo foi aplicado o MEEM.

Em seguida, foram realizados os testes TUG e SPPB. Para realização do teste TUG foi solicitado que, após o comando verbal do avaliador, a voluntária se levantasse de uma cadeira de $46 \mathrm{~cm}$ de altura, caminhasse o mais rápido possível por 3 metros, girasse $180^{\circ}$, retornasse e sentasse novamente na cadeira (SHUMWAY-COOK et al., 2000). $\mathrm{O}$ cronômetro foi disparado ao primeiro movimento do tronco da voluntária e a mensuração da duração do teste foi finalizada quando a mesma posicionava o tronco no encosto da cadeira. Para realização do teste SPPB as voluntárias foram solicitadas inicialmente a permanecer por 10 segundos em cada uma das seguintes posições: em pé com os pés juntos, em pé com um pé parcialmente à frente e em pé com um pé totalmente à frente. Posteriormente, foram avaliados o tempo necessário para caminhar uma distância de 3 ou 4 metros utilizando passo e calçados habituais e o tempo necessário para levantar e sentar cinco vezes consecutivas de uma cadeira sem o auxílio dos membros superiores. A pontuação para cada teste varia numa escala de zero (pior desempenho) a quatro pontos (melhor desempenho), totalizando uma pontuação máxima final variando de 0 a 12 pontos (NAKANO, 2007).

\section{Análise estatística}

As análises estatísticas foram processadas no programa SPSS, versão 15.0. Foram realizadas análises descritivas utilizando medidas de frequência e porcentagem, tendência central (média) e de variabilidade (amplitude e desvio-padrão) das características clínicas e sóciodemográficas, das quedas e dos desempenhos nos testes cognitivos e funcionais. A normalidade da distribuição dos dados foi analisada utilizando o teste Kolmogorov-Smirnov. As diferenças de médias das variáveis principais entre os dois grupos de idosas foram analisadas utilizando o teste $t$-student para amostras independentes. Para verificar a correlação entre idade, tempo de participação em atividades comunitárias, desempenho cognitivo e funcional foi utilizado o teste de correlação de Pearson. O nível de significância $(\alpha)$ de 0,05 foi considerado.

\section{RESULTADOS}

\section{Características das participantes}

As características clínicas e demográficas das idosas estão descritas na Tabela 1. As idosas participantes de atividades comunitárias ligadas à igreja apresentaram um tempo médio de participação de 23,62 anos, média de idade significativamente menor $(70,40 \pm 7,34$ anos vs $77,33 \pm 6,26$ anos) e estudaram significativamente mais anos $(9,42 \pm 5,87$ vs $3,34 \pm 2,28)$ que as idosas não-participantes. Quanto ao estado civil, a maioria das idosas participantes de atividades comunitárias era casada (50\%) ou viúva $(40 \%)$, enquanto a maioria das não-participantes era viúva $(55,6 \%)$. Com relação às doenças auto-relatadas, os dois grupos apresentaram alta prevalência de hipertensão arterial sistêmica, vestibulopatia e osteoporose, entretanto o grupo das idosas não-participantes apresentou percentuais mais elevados desses diagnósticos, além de apresentar alta prevalência de relato de diabetes e osteoartrose.

\section{Características das quedas}

As idosas participantes de atividades comunitárias apresentaram menor prevalência de quedas auto-relatadas quando comparadas às não-participantes (Tabela 2). Dentre as idosas que referiram quedas, observou-se, no grupo de não-participantes de atividades ligadas à igreja, maior repercussão funcional, maior tempo de permanência no chão, maior necessidade de ajuda para levantar após a queda. Adicionalmente, a maioria das idosas não-participantes de atividades referiu medo de cair (66,7\%) enquanto a maioria das participantes $(70 \%)$ negou esse sentimento. 
OliveIRA, A. M., GARCIA, P. A. Perfil demográfico. Rev. Ter. Ocup. Univ. São Paulo, v. 22, n. 2, p. 153-161, maio/ago. 2011.

Tabela 1. Características Clínicas e Demográficas da Amostra

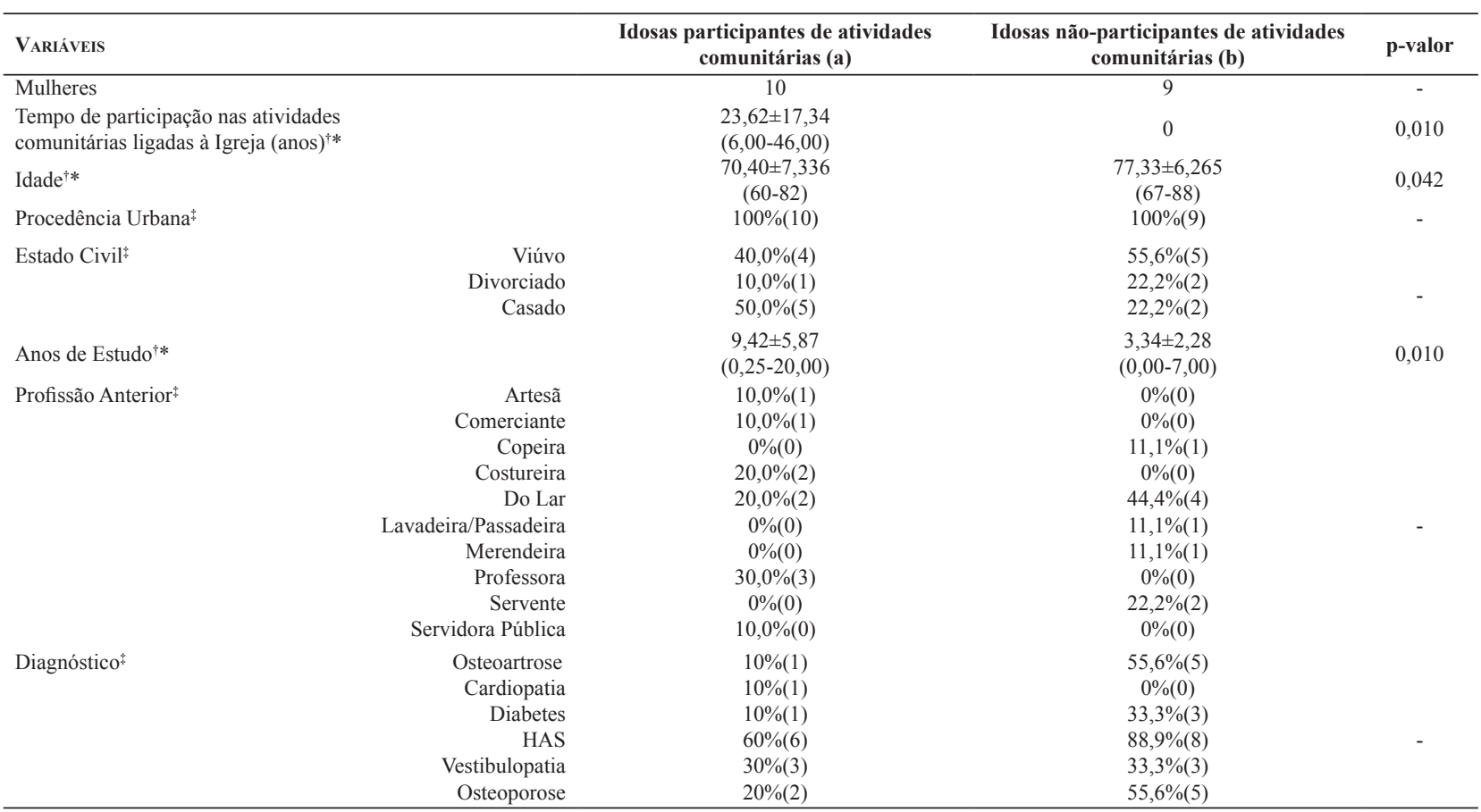

†média ( \pm DP) e amplitude (mínimo-máximo); ${ }^{\ddagger}$ proporção (n); *p<0,05 (teste t student para amostras independentes).

Tabela 2. Características das quedas referidas pelas idosas da amostra

\begin{tabular}{|c|c|c|c|}
\hline VARIÁVEIS & $\begin{array}{l}\text { Idosas participantes de atividades comu- } \\
\text { nitárias (a) }\end{array}$ & $\begin{array}{c}\text { Idosas não-participantes de ativida- } \\
\text { des comunitárias (b) }\end{array}$ & p-valor \\
\hline Mulheres & 10 & 9 & - \\
\hline Relato de quedas anteriores ${ }^{\ddagger}$ & $20,0 \%(2)$ & $33,3 \%(3)$ & - \\
\hline Número de Quedas ${ }^{\dagger}$ & $\begin{array}{l}1,00 \pm 0,000 \\
\quad(1-1)\end{array}$ & $\begin{array}{l}3,00 \pm 2,828 \\
\quad(1-5)\end{array}$ & 0,423 \\
\hline \multicolumn{4}{|l|}{ Repercussão funcional ${ }^{*}$} \\
\hline $\begin{array}{l}\text { Não } \\
\text { Sim }\end{array}$ & $\begin{array}{c}100,0 \%(2) \\
0 \%(0)\end{array}$ & $\begin{array}{l}33,3 \%(1) \\
66,7 \%(2)\end{array}$ & \multirow[t]{2}{*}{-} \\
\hline Causas ${ }^{\ddagger}$ & & & \\
\hline $\begin{array}{l}\text { Ausência de motivo aparente } \\
\text { Ambiental }\end{array}$ & $\begin{array}{c}0 \%(0) \\
100,0 \%(2)\end{array}$ & $\begin{array}{l}33,3 \%(1) \\
66,7 \%(2)\end{array}$ & - \\
\hline $\begin{array}{l}\text { Local da Queda } \\
\text { No domicílio } \\
\text { Fora do domicílio }\end{array}$ & $\begin{array}{l}50,0 \%(1) \\
50,0 \%(1)\end{array}$ & $\begin{array}{l}66,7 \%(2) \\
33,3 \%(1)\end{array}$ & - \\
\hline \multicolumn{4}{|l|}{ Para que lado caiu } \\
\hline Anterior & $100,0 \%(2)$ & $33,3 \% \%(1)$ & \multirow[b]{2}{*}{-} \\
\hline $\begin{array}{l}\text { Lateral } \\
\text { Posterior }\end{array}$ & $\begin{array}{l}0 \%(0) \\
0 \%(0)\end{array}$ & $\begin{array}{c}0 \%(0) \\
33,3 \%(1)\end{array}$ & \\
\hline $\begin{array}{l}\text { Posterior } \\
\text { Tempo de Permanência no chão }{ }^{\dagger} \\
\text { Necessidade de ajuda para levantar }\end{array}$ & $\begin{array}{c}0 \%(0) \\
0,00 \pm 0,000 \\
(0-0)\end{array}$ & $\begin{array}{c}33,3 \%(1) \\
10,00 \pm 17,321 \\
(0-30)\end{array}$ & 0,495 \\
\hline Não & $100,0 \%(2)$ & $66,7 \%(2)$ & \multirow{2}{*}{-} \\
\hline Sim & $0 \%(0)$ & $33,3 \%(1)$ & \\
\hline $\begin{array}{l}\text { Nega Fratura em decorrência das quedas } \\
\text { Medo de cair }\end{array}$ & $100 \%(2)$ & $100 \%$ (3) & - \\
\hline Não & $70,0 \%(7)$ & $33,3 \%(3)$ & - \\
\hline Sim & $30,0 \%(3)$ & $66,7 \%(6)$ & - \\
\hline \multicolumn{4}{|l|}{ Hábitos de Risco* } \\
\hline Não & $60,0 \%(6)$ & $66,7 \%(6)$ & - \\
\hline Sim & $40,0 \%(4)$ & $33,3 \%(3)$ & - \\
\hline
\end{tabular}

†média ( \pm DP) e amplitude (mínimo-máximo); ${ }^{\ddagger}$ proporção; *p<0,05 (teste t student para amostras independentes). 
OLIVEIRA, A. M., GARCIA, P. A. Perfil demográfico. Rev. Ter. Ocup. Univ. São Paulo, v. 22, n. 2, p. 153-161, maio/ago. 2011.

\section{Desempenho cognitivo e funcional das idosas}

As idosas que participam de atividades comunitárias ligadas à igreja apresentaram desempenhos cognitivos
(MEEM) e funcionais (TUG e SPPB) significativamente melhores que as que não participam dessas atividades (Tabela 3).

Tabela 3. Desempenho cognitivo e funcional das idosas da amostra

\begin{tabular}{|c|c|c|c|}
\hline VARIÁVEIS & $\begin{array}{c}\text { Idosas participantes de } \\
\text { atividades comunitárias (a) }\end{array}$ & $\begin{array}{l}\text { Idosas não-participantes de ativi- } \\
\text { dades comunitárias (b) }\end{array}$ & p-valor \\
\hline MEEM (pontuação) $)^{\dagger *}$ & $\begin{array}{c}25,70 \pm 3,368 \\
(20-30)\end{array}$ & $\begin{array}{l}20,00 \pm 4,873 \\
(13-26)\end{array}$ & 0,008 \\
\hline TUG (segundos) $)^{\dagger *}$ & $\begin{array}{c}7,60 \pm 1,506 \\
(5-9)\end{array}$ & $\begin{array}{c}10,33 \pm 3,240 \\
(7-18)\end{array}$ & 0,028 \\
\hline Pontuação SPPB Pés juntos ${ }^{\dagger}$ & $\begin{array}{l}1,00 \pm 0,000 \\
(1-1)\end{array}$ & $\begin{array}{l}1,00 \pm 0,000 \\
(1-1)\end{array}$ & \\
\hline Pontuação SPPB Pé Parcialmente à frente ${ }^{\dagger}$ & $\begin{array}{l}1,00 \pm 0,000 \\
(1-1)\end{array}$ & $\begin{array}{l}0,78 \pm 0,441 \\
(0-1)\end{array}$ & 0,169 \\
\hline Pontuação SPPB Pé Totalmente à frente ${ }^{\dagger}$ & $\begin{array}{c}1,70 \pm 0,483 \\
(1-2)\end{array}$ & $\begin{array}{c}1,11 \pm 0,782 \\
(0-2)\end{array}$ & 0,062 \\
\hline Pontuação Total do Equilíbrio ${ }^{\dagger}$ & $\begin{array}{c}3,70 \pm 0,483 \\
(3-4)\end{array}$ & $\begin{array}{c}3,11 \pm 1,167 \\
(1-4)\end{array}$ & 0,188 \\
\hline Pontuação Tempo de Marcha ${ }^{\dagger}$ & $\begin{array}{c}1,20 \pm 0,422 \\
(1-2)\end{array}$ & $\begin{array}{l}1,00 \pm 0,000 \\
(1-1)\end{array}$ & 0,168 \\
\hline Pontuação Levantar e Sentar da Cadeira ${ }^{\dagger *}$ & $\begin{array}{c}3,30 \pm 1,252 \\
(1-4)\end{array}$ & $\begin{array}{c}2,00 \pm 1,414 \\
(0-4)\end{array}$ & 0,048 \\
\hline Pontuação Total SPPB ${ }^{\dagger *}$ & $\begin{array}{c}8,20 \pm 1,476 \\
(5-9)\end{array}$ & $\begin{array}{c}6,11 \pm 2,369 \\
(2-9)\end{array}$ & 0,032 \\
\hline
\end{tabular}

†média ( \pm DP) e amplitude (mínimo-máximo); ${ }^{*} \mathrm{p}<0,05$ (teste t student para amostras independentes).

Correlação entre o tempo de participação de atividades comunitárias de igreja, idade, desempenho cognitivo e funcional nas idosas

$\mathrm{Na}$ análise de associação (Tabela 4), observouse correlação significativa do tempo de participação de atividades comunitárias ligadas à igreja com a idade $(\mathrm{r}=-0,579 ; \mathrm{p}=0,009)$ e com o desempenho cognitivo $(\mathrm{r}=0,467 ; \mathrm{p}=0,044)$. Adicionalmente, o desempenho cognitivo correlacionou-se significativamente com a idade $(\mathrm{r}=-0,579 ; \mathrm{p}=0,009)$ e com o desempenho funcional no TUG $(\mathrm{r}=-0,473 ; \mathrm{p}=0,041)$ e no SPPB $(\mathrm{r}=0,661 ; \mathrm{p}=0,005)$, e o desempenho no SPPB correlacionou-se significativamente com o desempenho no TUG $(r=-0,720 ; p=0,001)$.

Tabela 4. Correlação entre as variáveis tempo de participação em atividade comunitária, idade, desempenho cognitivo e funcional nas idosas

\begin{tabular}{|c|c|c|c|c|c|}
\hline Variáveis & $\begin{array}{l}\text { Participação em ativida- } \\
\text { de comunitária (anos) }\end{array}$ & Idade & MEEM & TUG & SPPB \\
\hline Participação em atividade comunitária (anos) & 1 & $-0,579 * *$ & $0,467 *$ & $-0,151$ & 0,146 \\
\hline Idade & & 1 & $-0,579 * *$ & 0,400 & $-0,422$ \\
\hline MEEM & & & 1 & $-0,473 *$ & $0,611 * *$ \\
\hline TUG & & & & 1 & $-0,720 * *$ \\
\hline SPPB & & & & & 1 \\
\hline
\end{tabular}

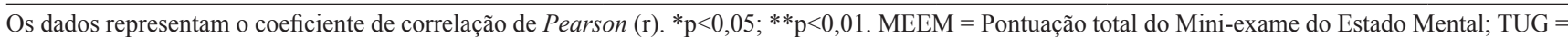
tempo no Teste Timed Get up and Go; SPPB = pontuação total no Short Physical Performance Battery. 


\section{DISCUSSÃO}

Neste estudo foram traçadas as características sóciodemográficas, clínicas e funcionais de idosas participantes e não-participantes de atividades comunitárias ligadas à igreja e investigadas as associações das variáveis e diferenças dessas características entre os dois grupos. Apesar de existirem pesquisas anteriores que investigaram algumas dessas relações (BRAAM et al., 1997; DIAS et al., 2010), observou-se que estudos envolvendo idosos que participam de atividades comunitárias especificamente ligadas à igreja não são comuns.

Atividades aparentemente corriqueiras, como fazer ou receber visitas e freqüentar a igreja, são fatores associados com a capacidade funcional, tornam-se muito importantes e parecem traduzir a idéia de vida ativa e serem capazes de contribuir para a manutenção da funcionalidade (ROSA et al., 2003), redução da propensão ao isolamento social e solidão. Neste estudo, foram avaliadas 10 idosas participantes de atividades comunitárias ligadas à igreja que apresentaram um tempo médio de atuação nessas atividades de 23,62 anos, tempo expressivo para gerar impacto no bemestar e qualidade de vida das idosas participantes. Os dados sócio-demográficos coletados apresentaram um breve perfil do grupo de pessoas que mantém participação ativa fora do domicílio, caracterizando idosas mais jovens, com nível de escolaridade mais alto, sendo a maioria casada ou viúva. Santos et al. (2009) salientam que o hábito de freqüentar a igreja é uma atividade comum entre idosos, destacando, também, a participação das mulheres idosas mais jovens.

Com relação às quedas, verificou-se que as idosas participantes, contrastando com as não-participantes, caem menos, não apresentam repercussões funcionais decorrentes das quedas, permanecem menos tempo no chão após as mesmas e a minoria refere medo de cair. De acordo com Lopes et al. (2009), Martin et al. (2005) e Scheffer et al. (2008) as quedas e o sentimento de medo de cair podem gerar impacto significativo no desempenho funcional, com consequente alteração do equilíbrio, depressão, ansiedade, redução do contato social e restrição de atividades.

No presente estudo, observou-se que as idosas participantes de atividades comunitárias apresentaram melhor desempenho cognitivo e que o desempenho cognitivo apresentou correlação positiva com o desempenho funcional. Estes dados sugerem, assim como outros estudos (McGUIRE et al., 2006; BASSUK et al., 2000), que idosos com função cognitiva preservada têm estilo de vida mais saudáveis, são mais ativos e apresentam melhor capacidade funcional e maior adesão aos tratamentos propostos quando adoecem. Além disso, na análise de correlação observou-se que quanto maior o tempo de participação nas atividades comunitárias, melhor o desempenho cognitivo, corroborando achados da literatura (SANTOS et al., 2009) e sugerindo também o impacto positivo do estilo de vida ativo, com participação de atividades externas, na estimulação e preservação cognitiva dos idosos (HSU, 2007; AVLUND et al., 2004).

Os resultados sobre os fatores envolvidos na funcionalidade evidenciaram que as idosas participantes apresentam desempenho funcional melhor do que as não-participantes. Em comparação com as idosas não-participantes, as participantes foram significativamente mais rápidas no teste TUG e apresentaram desempenho significativamente melhor nos registros do SPPB. Esses achados corroboram o estudo de Bourne (2009) que indicaram seis fatores que explicam a capacidade funcional em idosos, dentre eles a freqüência à igreja. Entretanto, no presente estudo, não foram observadas correlações significativas entre o tempo de participação nas atividades de igreja e o desempenho nos testes funcionais. Desta forma, sugere-se que a manutenção da capacidade funcional é fundamental para que os idosos se mantenham independentes, participantes em atividades familiares e comunitárias (AVLUND et al., 2002; BROOKS et al., 2006; PODSIADLO; RICHARDSON, 1991; SHUMWAY-COOK et al., 2000), porém a conservação de um estilo de vida ativo com participação em atividades comunitárias não é suficiente para prevenir declínios funcionais e risco de queda. Assim, torna-se necessário planejar e associar programas de treinamentos específicos para melhora funcional e prevenção de quedas às habituais atividades comunitárias ligadas à igreja realizadas pelos grupos de idosos (NITZ; CHOY, 2004; OSTIR et al., 2002).

Com o delineamento de natureza transversal deste estudo as inferências em relação ao impacto da participação nas atividades comunitárias de igreja no desempenho cognitivo e funcional dos idosos são restritas. Pode-se questionar a influência dos recursos econômicos, das incapacidades funcionais, falta de acesso a transporte e/ou das barreiras ambientais na participação dos idosos nessas atividades (ADAMS; BRITTAIN, 1988), e não apenas a influência da participação nos desfechos avaliados. Entretanto, este desenho de estudo e a análise de correlação provêem a caracterização dos idosos atuantes nas atividades comunitárias ligadas à igreja, possibilitam o incentivo destas práticas entre os grupos de idosos e reafirmam a necessidade de mudança no paradigma de atenção à saúde da população idosa, com novas estratégias, que substituam os modelos tradicionais centrados na assistência hospitalar e/ou asilar (DIAS et al., 2010). Desta forma, estes achados contribuem, mesmo que de forma primária, para elaboração de programas destinados a facilitar a participação dos idosos nas 
atividades da igreja e de programas complementares, tais como oficinas de memória, informática, lazer e de atividades manuais, de baixo custo e fácil implementação, que possam ser associados às atividades comunitárias de igreja, visando potencializar os efeitos positivos destas atividades, prevenir morbidades e melhorar a qualidade de vida e bem-estar dos idosos (CASTRO et al., 2007), contribuindo com as políticas públicas e assegurando a inclusão social e a atenção integral à saúde do idoso.

\section{CONCLUSÕES}

Os resultados deste estudo possibilitaram descrever as características sócio-demográficas, clínicas e funcionais de idosas participantes e não-participantes de atividades comunitárias ligadas à igreja, indicando que as idosas participantes apresentaram um perfil favorável ao envelhecimento ativo, incluindo melhor desempenho funcional e cognitivo. Desta forma, sugere-se que a participação em atividades comunitárias ligadas à igreja exerça influência positiva na manutenção da capacidade funcional e cognitiva, no envelhecimento ativo e saudável, porém deve ser incentivada sua associação a programas de educação em saúde, prática de atividades recreativas, físicas e culturais. Adicionalmente, estes resultados podem contribuir para a reflexão sobre a importância dos aspectos sociais, produtivos e de lazer no envelhecimento, para o planejamento de novas estratégias em saúde e nas futuras discussões sobre as dificuldades de abordagem de idosas inativas e não-participantes de atividades comunitárias.

OLIVEIRA, A. M., GARCIA, P. A. Demographic, clinical and functional profile of elderly participants and non-participants in community activities of church. Rev. Ter. Ocup. Univ. São Paulo, v. 22, n. 2, p. 153-161, maio/ago. 2011.

\begin{abstract}
Introduction: the social and community activities are referred in scientific literature as strategies to promote health and quality of life during aging. Objective: To describe and compare the demographic, clinical and functional status of participants and non-participants elderly in community activities of the church. Methods: Nineteen elderly, being 10 participants and 9 non-participants in church activities, underwent socio-demographic, cognitive (Mini-Mental State Examination) and functional (Timed Get Up and Go and Short Physical Performance Battery) assessment. Results: The elderly participants in these activities had an average time of 23.62 years of activity. These elderly were younger age, more years of education, better cognitive performance, lower prevalence of self-reported falls and fear of falling, and better functional performance when compared to nonparticipants. There was a significant correlation between years of participation in activities with age $(r=-0.579)$ and with cognitive performance $(r=0.467)$. Conclusion: The elderly participants in church activities showed clinical and demographic profile more favorable for active aging and better cognitive and functional performance when compared to non-participants. Participation in these activities seems to be beneficial for maintaining the functional capacity, to have active and healthy aging, and thins this way should be encouraged to complement the programs of health promotion and disease prevention.
\end{abstract}

KEY WORDS: Aged; Activities of daily living; Cognition; Consumer participation; Health promotion; Mobility limitation.

\title{
REFERÊNCIAS
}

ADAMS, R. G.; BRITTAIN, J. L. Functional status and church participation of the elderly: theoretical and practical implications. J. Relig. Gerontol., v. 33, n. 4, p. 35-48, 1988.

AVLUND, K.; DAMSGAARD, M. T.; SAKARI-RANTALA, R.; LAUKKANEM, P.; SCHROLL, M. Tiredness in daily activities among nondisabled old peolpe as determinant of onset of disability. J. Clin. Epidemiol., v. 55, p. 865-973, 2002.
AVLUND, K.; LUND, R.; HOLSTEIN, B. E.; DUE, P.; SAKARIRANTALA, R.;HEIKKINEN, R. L. The impact of structural and functional characteristics of social relations as determinants of functional decline. J.Gerontol.B Psychol.Sci.Soc.Sci., v. 59, n. 1, p. S44-S51, 2004.

BASSUK, S. S.; WYPIJ, D.;BERKMAN, L. F. Cognitive impairment and mortality in the community-dwelling elderly. 
Am. J. Epidemiol., v. 151, n. 7, p. 676-688, 2000.

BERTOLUCCI, P. H.; BRUCKI, S. M.; CAMPACCI, S. R.;JULIANO, Y. O mini-exame do estado mental em uma população geral: impacto da escolaridade. Arq. Neuropsiquiatr., v. 52 , n. 1 , p. 1-7, 1994.

BOURNE, P. A. Activities of daily living, instrumental activities for daily living and predictors of functional capacity of older men in Jamaica. North Am. J. Med. Sci., v. 1, n. 4, p. 184-192, 2009.

BRAAM, A. W.; BEEKMAN, A. T.; DEEG, D. J.; SMIT, J. H.;VAN, T. W. Religiosity as a protective or prognostic factor of depression in later life; results from a community survey in The Netherlands. Acta Psychiatr. Scand., v. 96, n. 3, p. 199-205, 1997.

BROOKS, D.; DAVIS, A. M.; NAGLIE, G. Validity of 3 physical performance measures in inpatient geriatric rehabilitation. Arch. Phys. Med. Rehabil., v. 87, n. 1, p. 105-110, 2006.

CARVALHO JAM, G. R. O envelhecimento da população brasileira: um enfoque demográfico. Cad Saúde Pública, v. 19, n. 3, p. 725-733, 2003.

CASTRO, P. C.; TAHARA, N.; REBELATTO, J. R.; DRIUSSO, P.; AVEIRO, M. C.;OISHI, J. Influência da universidade aberta da terceira idade (UATI) e do programa de revitalização (REVT) sobre a qualidade de vida de adultos de meia-idade e idosos. Rev. Bras. Fisioter., v. 11, n. 6, p. 461-467, 2007.

COSTA, E. F. A.; PORTO, C. C.;SOARES, A. T. Envelhecimento Populacional brasileiro e o aprendizado de geriatria e Gerontologia. Revista da UFG - Tema melhor idade, v. 5, n. 2, 2003.

DIAS, E. G.; DUARTE, Y. A. O.;LEBRÃO, M. L. Efeitos longitudinais das atividades avançadas de vida diária em idosos: implicações para a reabilitação gerontológica. Mundo Saúde, v. 34 , n. 2, p. 258-267, 2010.

FRIED, L. P.; FERRUCCI, L.; DARER, J.; WILLIAMSON, J. D.; ANDERSON, G. Untangling the concepts of disability, frailty, and comorbidity: implications for improved targeting and care. $J$. Gerontol. A Biol. Sci. Med. Sci., v. 59, n. 3, p. 255-263, 2004.

FRIED, L. P.; TANGEN, C. M.; WALSTON, J.; NEWMAN, A. B.; HIRSCH, C.; GOTTDIENER, J. et al. Frailty in older adults: evidence for a phenotype. J. Gerontol. A Biol. Sci. Med. Sci., v. 56, n. 3, p. M146-M156, 2001.

GARRIDO R. ; MENEZES P.R. O Brasil está envelhecendo: boas e más notícias por uma perspectiva epidemiológica. Rev. Bras. Psiquiatr., v. 24, supl. I, p. 3-6, 2002.

HECKMAN, P. R. W. O idoso frágil. In: FREITAS, E. V.; PY L.; CANÇADO, F. A. X. (Eds.). Tratado de geriatria e gerontologia. Rio de Janeiro: Guanabara Koogan, 2006. p. 926-929.
HSU, H. C. Does social participation by the elderly reduce mortality and cognitive impairment? Aging Ment. Health, v. 11, n. 6, p. $699-707,2007$.

KILSZTAJN, S.; ROSSBACH, A.; CÂMARA, M. B.;CARMO, M. S. M. Serviços de saúde, gastos e envelhecimento da população brasileira. Rev. Bras. Est. Pop., v. 20, n. 1, p. 93-108, 2003.

LOPES, K. T.; COSTA, D. F.; SANTOS, L. F.; CASTRO, D. P.; BASTONE, A. C. Prevalência de medo de cair em uma população de idosos da comunidade e sua correlação com mobilidade, equilíbrio dinâmico, risco e histórico de quedas. Rev. Bras. Fisioter., v. 13, n. 3, p. 223-229, 2009.

MARTIN, F. C.; HART, D.; SPECTOR, T.; DOYLE, D. V.; HARAR, D. Fear of falling limiting activity in young-old women is associated with reduced functional mobility rather than psychological factors. Age Ageing, v. 34, n. 3, p. 281-287, 2005.

McGUIRE, L. C.; FORD, E. S.;AJANI, U. A. The impact of cognitive functioning on mortality and the development of functional disability in older adults with diabetes: the second longitudinal study on aging. BMC Geriatr., v. 6, p. 8, 2006.

NAKANO, M. M. Versão Brasileira da Short Physical Performance Battery - SPPB: Adaptação cultural e estudo da confiabilidade. 2007. Faculdade de Educação, Universidade Estadual de Campinas, Campinas, 2007.

NITZ, J. C. ; CHOY, N. L. The efficacy of a specific balancestrategy training programme for preventing falls among older people: a pilot randomised controlled trial. Age Ageing, v. 33, n. 1, p. $52-58,2004$.

ORGANIZAÇÃOMUNDIALDE SAÚDE(OMS). Envelhecimento ativo: uma política de saúde. Brasília: Organização Pan-americana de Saúde, 2005.

OSTIR, G. V.; VOLPATO, S.; FRIED, L. P.; CHAVES, P.;GURALNIK, J. M. Reliability and sensitivity to change assessed for a summary measure of lower body function: results from the Women's Health and Aging Study. J. Clin. Epidemiol., v. 55, n. 9, p. 916-921, 2002.

PODSIADLO, D. ; RICHARDSON, S. The timed "Up \& Go": a test of basic functional mobility .

ROSA, T. E. C.; BENÍCIO, M. H. D.; LATORRE, M. R. D. O.;RAMOS, L. R. Fatores determinantes da capacidade funcional entre idosos. Rev. Saude Publica, v. 37, n. 1, p. 40-48, 2003.

SANTOS, P. L.; FORONI, P. M.;CHAVES, M. C. F. Atividades físicas e de lazer e seu impacto sobre a cognição no envelhecimento. Medicina (Ribeirão Preto), v. 42, n. 1, p. 54-60, 2009.

SCHEFFER, A. C.; SCHUURMANS, M. J.; VAN, D. N.; VAN DER HOOFT, T.;DE ROOIJ, S. E. Fear of falling: measurement strategy, prevalence, risk factors and consequences among older persons. Age Ageing, v. 37, n. 1, p. 19-24, 2008. 
OliveIRA, A. M., GARCIA, P. A. Perfil demográfico. Rev. Ter. Ocup. Univ. São Paulo, v. 22, n. 2, p. 153-161, maio/ago. 2011.

SHUMWAY-COOK, A.; BRAUER, S.; WOOLLACOTT, M. Predicting the probability for falls in community-dwelling older adults using the timed up \& go test. Phys. Ther., v. 80, n. 9, p. 896-903, 2000.

SILVA, T. A. A.; FRISOLI JÚNIOR, A.; PINHEIRO, M. M.;SZEJNFELD, V. L. Sarcopenia associada ao envelhecimento: aspectos etiológicos e opções terapêuticas. Rev. Bras. Reumatol., v. 46, n. 6, p. 391-397, 2006.

WALSTON, J.; HADLEY, E. C.; FERRUCCI, L.; GURALNIK,
J. M.; NEWMAN, A. B.; STUDENSKI, S. A.; et al. Research agenda for frailty in older adults: toward a better understanding of physiology and etiology: summary from the American Geriatrics Society/National Institute on Aging Research Conference on Frailty in Older Adults. J. Am. Geriatr. Soc., v. 54, n. 6, p. 9911001, 2006.

WONG, L. L. R.; CARVALHO, J. A. O rápido processo de envelhecimento populacional no Brasil: sérios desafios para as políticas públicas. Rev. Bras. Est. Pop., v. 23, n. 1, p. 5-26, 2006.

Recebido para publicação: 15/04/2011

Aceito para publicação: 15/05/2011 